\title{
Occurrence of polyglucosan bodies in temporal lobe epilepsy
}

\author{
Hugues Loiseau, Cécile Marchal, Anne Vital, Claude Vital, Alain Rougier, Pierre Loiseau
}

\begin{abstract}
Massive occurrence of polyglucosan bodies (PBs) was found within the surgically removed temporal lobe of a 34 year old woman with complex partial seizures. This peculiar feature is very unusual in neuropathological examinations of epileptogenic foci. This patient could not be included in any of the classic diseases in which PBs are found. She exhibited a localised form of glycogen storage disease.
\end{abstract}

$(\mathcal{F}$ Neurol, Neurosurg Psychiatry 1992;55:1092-1093)

Polyglucosan bodies (PBs) are found in various pathological conditions and in normal ageing. The neuropathology of temporal lobe epilepsy is described in many papers and textbooks. ${ }^{1-5}$ $\mathrm{PBs}$ have not figured prominently in those descriptions.

\section{Case report}

A 34 year old female presented with intractable complex partial seizures with an onset at 13 years of age. Neurological examination was normal. Some EEGs showed a right temporal spike focus and others were normal. CT scan was normal. MRI showed an increased signal intensity on $\mathrm{T} 2$-weighted images in the medial part of the right temporal lobe. SPECT with $99 \mathrm{~m}$ Tc HMPAO was normal. SEEG recorded seizures arising in the medial right temporal lobe. At operation in June 1988, Ammon's horn was swollen and tough on palpation. Uncinate herniation was noted. An anterior temporal lobectomy and amygdalohippocampectomy was realised. Fragments of the anterior temporal region, amygdala and hippocampus were examined pathologically. On paraffin-embedded fragments there was a severe neuronal loss in the pyramidal layer of the Ammon's horn and a few scattered PBs. These PBs were very numerous in the subcortical white matter (fig, left) and in the most superficial areas of the cortex, mainly in a perivascular location. These modifications were also present in the adjacent neocortex. No PBs were seen in neuronal bodies. On ultrastructural examination (fig, right), these PBs were located between myelinated fibres and astrocytic processes. They were not shown in an intra-axonal location. These rounded bodies were composed of intermingled filaments. Muscle and nerve were normal. Drugs were withdrawn one year after surgery. The patient remains seizure-free after surgery.

\section{Discussion}

The term of PB was proposed to group Lafora bodies (LB), lafora-like bodies, corpora amylacea and Bielschowsky bodies on the basis of their biochemical similitude. ${ }^{6} \mathrm{~A}$ rigorous histochemical differentiation between these different anomalies appears impossible. ${ }^{67}$ Both LB and corpora amylacea are mainly made up of glucose polymers, that is, polyglucosans. PB are derived from glycogen. Accumulation of glycogen in the brain reflects a disordered turnover that can result from two mechanisms: increased synthesis or decreased degradation. ${ }^{7}$ Errors in the metabolism of glycogen are frequent but few of them lead to PB formation. ${ }^{8}$ The pathway leading from glycogen accumulation to PB formation is still unknown.

PB are a hallmark of two diseases: Lafora disease (progressive myoclonic epilepsy) and adult polyglucosan disease (progressive lower and upper motor neuron involvement, neurogenic bladder and sometimes dementia). In both conditions PB are present in the perikarya of cerebral neurons, but not in glial cells. They are also found in peripheral nerves and various organs. $^{6911}$

PB have occasionally been reported in rare cases of a variety of neurological diseases: olivopontocerebellar degeneration, amytrophic lateral sclerosis, Werner syndrome, hepatic encephalopathy, Shy-Drager syndrome, Pelizaeus-Merzbacher syndrome, pallido-nigroluysial atrophy and in Andersen disease. In all the cases they were located within the neurons. This site characterises Lafora bodies.

Bielschowsky bodies are also intraneuronal inclusions but restricted to neurons of the external pallidum. ${ }^{12}$ They are observed in cases of choreoathetosis and cerebral palsy.

Conversely, corpora amylacea, the third form of $\mathrm{PB}$, occur predominantly in the astroglia. They have long been recognised as occurring routinely and non-specifically in the CNS during the course of ageing. ${ }^{13}$ Corpora amylacea in the hippocampus were observed using electron microscopy within astrocytic processes. ${ }^{14}$ They were also found, to a lesser extent, within neurons. ${ }^{13}$

The pathognomonic significance of these structures depends on their regional distribution: Corpora amylacea occur predominantly in the astroglia, LB occur typically in the 

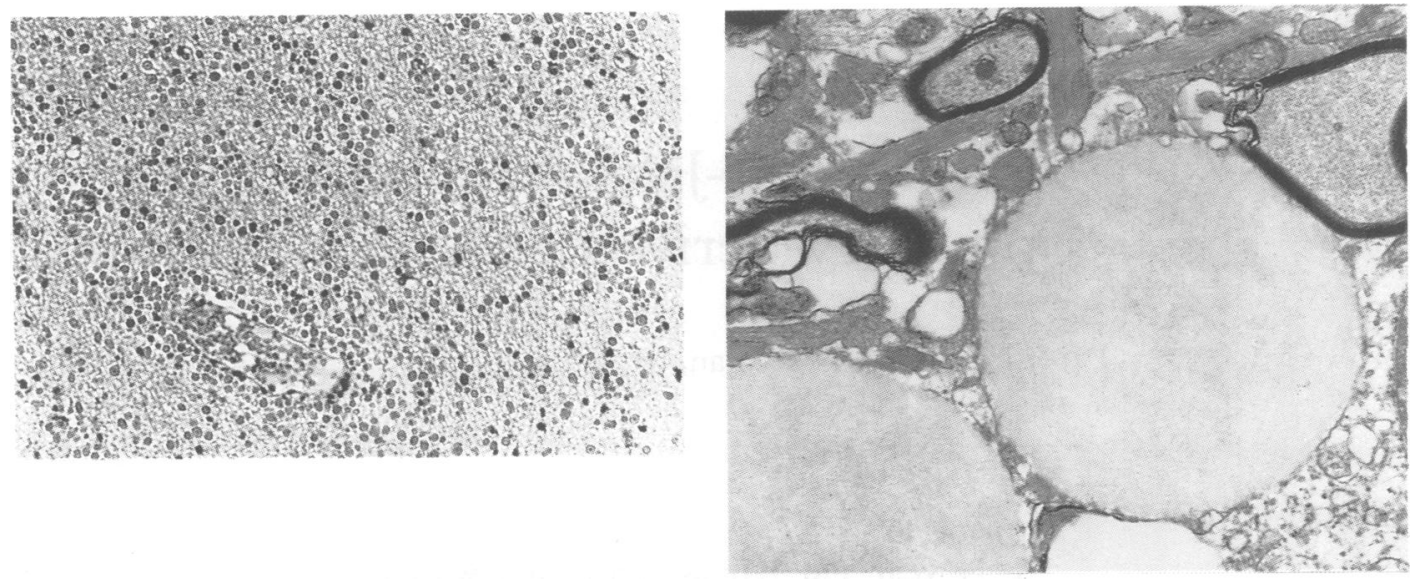

Figure Left: There are numerous polyglucosan bodies in the temporal white matter (hematoxylin-eosin; original magnification, $\times 210$ ); Right: Electron micrograph of two PB. They are located near myelinated fibres and astrocytic processes (original magnification, $\times 5$ ).

neurons, and Bielschowsky bodies are restricted to the neurons of the external pallidum. Throughout these various aetiologies one point should be underlined: the ressemblance does not imply a common aetiology for all conditions in which such bodies occur; it probably signifies only their sharing of the final stretch along their causative pathway. ${ }^{8}$

Our patient cannot be included in any of the classical diseases in which PBs are found. She exhibited a localised form of glycogen storage disease. In our series of 85 temporal resections, pathology revealed a few PBs in 4 patients, but not as a prominent feature. An extensive search throughout the literature concerning the neuropathology of localisation-related epilepsies provided only one similar case. ${ }^{14}$ Two other cases were found by serendipity among material for biochemical or electron microscopy studies of corpora amylacea without comment on their possible clinical significance. ${ }^{1516}$

PB accumulation may be interpreted as a consequence or cause of the patient's epilepsy, or unrelated to it.

Glycogen accumulation is known to occur within the brain in various conditions such as ischaemic or anoxic events and under the influence of several drugs. ${ }^{8}$ A temporal uncus herniation was present and may be the result of seizure-induced brain oedema. However, temporal herniation is commonly found in temporal lobe epilepsy, and, to our knowledge, never associated with PB storage. In a similar manner, long-lasting medical treatments are usual in epileptic candidates for surgery and do not lead to PB storage.

PB have been observed in various disorders having in common a gliosis which is the expression of proliferative activities by fibrillary astrocytes. It has been suggested that gliosis may trigger the deposition of $\mathrm{PB}{ }^{8}$ Gliosis is a common feature observed in temporal lobe epilepsy. But PB deposition is not.

Proliferation of astrocytes in response to epileptic seizures could yield PB accumulation or a proliferative activity in increasing metabolic demand precipitate the manifestations of a latent enzymatic deficiency. A massive accumulation of $\mathrm{PB}$ in astrocytic processes might impair the exchange of essential metabolites between the parenchyma and the vessels and might impair the normal functioning of the cells. In our patient, as in the Australian patient, ${ }^{14}$ unusually large numbers of PBs were the only significant abnormality. They may be interpreted as the origin of an epileptic focus, the removal of which rendered the patients seizure-free.

1 Engel J Jr, Driver GV, Falconer MA. Electrophysiological correlates of pathology and surgical results in temporal obe epilepsy. Brain 1975;98:129-36.

2 Mathieson G. Pathology of temporal lobe foci. In: Penry JK Daly DD, eds. Advances in neurology, Vol II; complex partial seizures and their treatment. New York: Raven Press, 1975:163-82.

3 Jensen I, Klinken L. Temporal lobe epilepsy and neuropathology. Acta Neurol Scand 1976;54:391-414.

4 Babb TL, Brown WJ. Pathological findings in epilepsy. In: Engel J Jr, ed. Surgical treatment of the epilepsy. New York: Raven Press, 1987:511-40.

5 Bruton CJ. The neuropathology of temporal lobe epilepsy. (Maudsley monography 31). Oxford: Oxford University Press, 1988.

6 Robitaille Y, Carpenter S, Karpati G, Dimauro S. A distinct form of adult polyglucosan body disease with massive involvement of central and peripheral neuronal processes involvement of central and peripheral neuronal processes
and astrocytes. A report of four cases and a review of the and astrocytes. A report of four cases and a review of the
occurrence of polyglucosan bodies in other conditions occurrence of polyglucosan bodies in other conditions
such as Lafora's disease and normal ageing. Brain such as Lafora's

7 Palmucci L, Anzil AP, Luh S. Intra-astrocytic glycogen granules and corpora amylacea stain positively for polyglucosans: a cytochemical contribution on the fine structural polymorphism of particulate polysaccharides. Acta Neuropathol 1982;57:99-102.

8 Austin J, Sakai M. Disorders of glycogen and related macromolecules in the nervous system. In: Vinken PJ,
Bruyn GW, eds. Handbook of clinical neurology. Amsterdam: North Holland, 1976:169-219.

9 Busard HLSM, Renier WO, Gabreëls FJM, Jaspar HHJ, Slooff JL, Janssen AJM, Van Haelst UJG. Lafora disease: a quantitative morphological and biochemical study of the cerebral cortex. Clin Neuropathol 1987;6:1-6.

10 Carpenter S, Karpati G. Sweat gland duct cells in Lafora disease: diagnosis by skin biopsy. Neurology 1981; 31:1564-8.

11 Gray F, Gherardi R, Marshall A, Janota I, Poirier J. Adult polyglucosan body disease (APBD). $\mathcal{f}$ Neuropathol Exp neurol 1988;47:459-74.

12 Yagishita S, Itoh Y, Nakano T, Amano N, Yokoi S, Hasegawa $\mathrm{O}$, Tanaka T. Pleomorphic intra-neuronal polyglucosan bodies mainly restricted to the pallidum. A case report. Acta Neuropathol 1983;62:159-63.

13 Anzil AP, Herrlinger H, Blinzinger $K$, Kronski D. Intraneuritic corpora amylacea: demonstration in orbital cortex of elderly subjects by means of early postmortem brain sampling and electron microscopy. Virchows Arch (A) 1974;364:297-301

14 Jackson GD, Berkovic SF, Tress BM, Kalnins RM, Fabinyi GCA, Bladin PF. Hippocampal sclerosis can be reliably detected by magnetic resonance imaging. Neurology 1990;40:1869-75.

15 Ramsey HJ. Ultrastructure of corpora amylacea. $7 \mathrm{Neu}$ ropathol Exp Neurol 1965;24:25-39.

16 Roukema PA, Oderkerk C. Isolation and preliminary characterization of corpora amylacea from human brain. Psychiat Neurol Neurochir 1970;73:87-96. 